\title{
Prognostic significance of miR-34a and its target proteins of FOXP1, p53, and BCL2 in gastric MALT lymphoma and DLBCL
}

\author{
Miaoxia He $\cdot$ Li Gao $\cdot$ Shimin Zhang $\cdot$ \\ Liyang Tao $\cdot$ Jianjun Wang $\cdot$ Jianmin Yang . \\ Minghua Zhu
}

Received: 27 June 2013/Accepted: 21 October 2013/Published online: 14 November 2013

(C) The International Gastric Cancer Association and The Japanese Gastric Cancer Association 2013

\begin{abstract}
Background Mucosa-associated lymphoid tissue (MALT) lymphoma and diffuse large B-cell lymphoma (DLBCL), which are the two most common types of gastric lymphomas, have different clinicopathological features and molecular characteristics with distinct clinical outcomes. Tumor suppressor miR-34a connects the p53 network with forkhead box protein 1 (FOXP1) and BCL2. Here, we investigated the prognostic value of these molecules in gastric MALT lymphoma and DLBCL for use in routine clinical practice.

Methods Relative miR-34a expression was detected by quantitative reverse transcriptase-polymerase chain reaction in 20 cases of MALT lymphomas and 20 cases of DLBCLs. Tissue microarray, in situ hybridization, and immunohistochemistry analysis were used to examine the expression of miR-34a and its regulated genes, FOXP1, p53, and BCL2 proteins, in 64 patients with gastric MALT lymphoma and in 58 patients with DLBCL. Helicobacter
\end{abstract}

Electronic supplementary material The online version of this article (doi:10.1007/s10120-013-0313-3) contains supplementary material, which is available to authorized users.

M. He · L. Gao · L. Tao · J. Wang · M. Zhu ( $\bowtie)$

Department of Pathology, Changhai Hospital, The Second

Military Medical University, 168 Changhai Rd.,

Shanghai 200433, China

e-mail: mhzhu2000@ hotmail.com

\section{S. Zhang}

Molecular Diagnosis Laboratories, Armed Forces Institute of Pathology, Washington, DC 20306, USA

J. Yang

Department of Hematology, Changhai Hospital, The Second Military Medical University, Shanghai 200433, China pylori infection, overall survival (OS), and progression-free survival (PFS) were documented.

Results The expression level of miR-34a was markedly decreased in MALT lymphomas and DLBCLs compared to normal gastric tissues and peripheral blood mononuclear cells. miR-34a was present in the cytoplasm and nucleus of lymphocytes. Its expression was significantly downregulated in MALT and DLBCL lymphoma tissues, as compared with normal lymphocytes. The expression level of miR-34a in DLBCL was lower than in MALT lymphoma. FOXP1 was found to be positive in $48 \%$, p53 in $20 \%$, and BCL2 in $68 \%$ of MALT lymphoma cases. The corresponding positive rates of these markers in DLBCL were 64,57 , and $52 \%$, respectively. High expression of FOXP1, p53, and BCL2 was seen in stage III and IV of both types of lymphomas. FOXP1, p53, and BCL2 positivity was associated with poor OS with both lymphoma types but OS with DLBCL was significantly lower than with MALT lymphoma.

Conclusions Decreased miR-34a expression and increased FOXP1, p53, and BCL2 coexpression to predict a poor OS for MALT lymphoma and DLBCL patients could become very important prognostic markers in daily clinical work. Further investigation of these changes may be of prognostic significance in clinical practice.

Keywords MALT lymphoma - DLBCL - Stomach · miR-34a $\cdot$ FOXP1/p53/BCL2 Prognosis

\section{Introduction}

Up to $40 \%$ of patients with non-Hodgkin lymphoma (NHL) present with an involvement of extranodal sites, which are usually in the gastrointestinal tract [1]. Primary 
gastrointestinal lymphomas are relatively common, with the large majority occurring in the stomach. In recent years, lymphoma has been identified as the second most common form of stomach malignant tumors [1, 2]. Among the gastric lymphomas, mucosa-associated lymphoid tissue (MALT) lymphoma and diffuse large B-cell lymphoma (DLBCL) are the two most common types [2].

Gastric MALT lymphoma and DLBCL have different clinicopathological features and molecular characteristics with distinct clinical outcomes [1-3]. Currently, there are no clear-cut pathological or molecular biomarkers of independent practical prognostic value for these two types of gastric lymphoma [1, 3, 4].

MicroRNAs (miRNAs) inhibit the transcription or translation of mRNA and have been shown to negatively regulate tumor suppressors or oncogenes, indicating that they may play a role in tumorigenesis [5]. Researchers suggest that a single or small number of genes play a major role in regulating miRNA-mediated genes and proteins [5, 6]. In the past few years, some studies suggest that miRNA-34a may act as a tumor suppressor by regulating apoptosis in several tumors [7]. It has also been shown that miR-34a-induced apoptosis is at least partly dependent on the presence of wild-type p53 genes, indicating that miR34a may feed back to p53 [8-11]. In similar studies, the status of p53 deletion or mutation was associated with miR-34a downregulation in prostate cancer, gastric cancer, and chronic B-cell lymphocytic leukemia [11, 12]. The same studies showed that BCL2 was targeted by miR-34a, and that there was a direct interaction between miR-34a and the tumor suppressor p53 and the oncogenic protein BCL2. This interaction was shown to increase the survival of miR-34a-expressing pro-B cells but did not significantly promote their differentiation [10, 11, 13, 14]. Other studies have shown that the forkhead box protein 1 (FOXP1) is involved in B-cell differentiation and survival [15]. The activity of FOXP1 may, therefore, be dependent on the downregulation of miR-34a, which allows B-cell development to proceed $[15,16]$. The effects of miR-34a on FOXP1 may provide a mechanism by which p53 suppresses tumor cells implying a connection between p53 and FOXP1 via the actions of miR-34a. In this manner, miR34a and FOXP1 are able to control the output of the B-cell developmental pathway. In differentiated B cells, FOXP1 appears to act as a B-cell oncogene [15, 16]. Its dysregulated expression in MALT lymphoma and amplification in many cases of DLBCL is thought to be the consequence of translocations to the immunoglobulin heavy locus $[17,18]$.

Taken together these findings suggest that miR-34a regulation of B-cell development and apoptosis is related to the tumor suppressor p53, oncogenic protein BCL2, and FOXP1. Thus, the present study investigated the role of miR-34a and its target proteins in two common gastric
B-cell lymphomas and evaluated possible associations between expression of miR-34a and target proteins on clinical outcomes.

\section{Materials and methods}

Patients and samples

Tumor samples from patients with gastric MALT lymphoma or DLBCL over a 10-year period (January 2000-December 2011) were retrieved from the archives of the Department of Pathology at the Changhai Hospital, the Second Military Medical University (Shanghai, China). Records from 122 patients (64 cases of MALT lymphoma and 58 cases of DLBCL) were included in the study. An additional 20 cases of MALT and 20 cases of DLBCL samples were collected from patients during surgical resections that had enough tumor tissue and stored in liquid nitrogen. Patient characteristics, clinical presentation, staging, laboratory findings, site and date of relapse, and survival were collected from hospital information systems. Patients had been evaluated using standard methods including history, physical examination, and biochemical-hematological tests. The Ann Arbor Staging System was used to determine disease stage. This study was approved by the institutional review board of Second Military Medical University, Shanghai, China. Signed informed consent was obtained from all study participants for sample collection and analysis.

\section{Morphological analysis and tissue microarray}

In all cases, tissue samples obtained by gastrectomy (containing distal, proximal, or local resection) were fixed in formalin and embedded in paraffin wax. Sections $4 \mu \mathrm{m}$ thick stained with hematoxylin and eosin were reviewed by three pathologists who graded the morphological features of MALT lymphoma and DLBCL according to the World Health Organization classification [1]. All slides were stained with toluidine blue stain to highlight the presence of Helicobacter pylori (HP).

Tissue microarrays (TMAs) were constructed by punching two tissue cylinders with a diameter of $0.8 \mathrm{~mm}$ from 122 patient archival paraffin blocks. The cylinders were transferred into a recipient paraffin block using a semiautomatic precision instrument (Beecher Instruments, Silver Spring, MD, USA).

Quantitative real-time reverse transcriptase-polymerase chain reaction (qRT-PCR) analysis

Frozen specimens of 20 MALT lymphoma and 20 DLBCL were selected from the 122 gastric lymphomas for miR-34a 
qRT-PCR analysis, The samples should have contained enough tumor tissues by macro-dissected methods using frozen sections. Three normal gastric tissue samples and two samples of peripheral blood mononuclear cells (PBMC) were selected as normal controls (NC).

Total RNA containing miRNA was extracted in 20 samples of MALT lymphoma and 20 samples of DLBCL, and 5 control samples were extracted using miRNeasy Mini Kit (Qiagen). cDNA was synthesized using miScript Reverse Transcription Kit (Qiagen) following the manufacturer's instructions. Reverse transcription was undertaken using $50 \mathrm{ng}$ total RNA with a primer specific for miR-34a, together with the SYBR Green microRNA reverse transcription kit. miRNAs were quantified using the SYBR Green miRNA qRT-PCR assay according to the manufacturer's protocol (Applied BioSystems). The qRTPCR reaction was carried out on a 7500 Fast Real-time System (Applied Biosystems). RNU6, a small RNA encoded in the intron of RPL5, was used for normalization in miRNA studies. All quantitative RT-PCRs were performed in triplicate. The data were analyzed using an automated baseline. The threshold cycle $\left(C_{\mathrm{t}}\right)$ was defined as the fractional cycle number at which the fluorescence exceeded the given threshold. The data obtained from the qRT-PCR were analyzed using the $\Delta \Delta C_{\mathrm{t}}$ method ( $2^{\Delta \Delta C_{\mathrm{t}}}$ ) [19]. The sequences of primers are listed in Supplementary Table 1.

miRCURY LNA miR-34a detection by in situ hybridization (ISH)

Sections $4 \mu \mathrm{m}$ thick were cut from microarray blocks. Locked nucleic acid in situ hybridization (LNA-ISH) was performed on gastric MALT and DLBCL lymphoma TMA using miRCURY LNA probes against Has-miR-34a (Exiqon, miR-34a LNA probe: 38487-05, Vedbaek, Denmark). Probes were used in accordance with manufacturer protocols. Lymphreactive hyperplasia was used for positive control.

Immunostaining procedures

Immunostaining was performed using mouse monoclonal antibodies against FOXP1 (JC12, 1:100; Abcam), p53 (1:100; Dako), and BCL2 (1:150; Dako). Routine monoclonal antibodies for tumor immunotypes including CD20 (1:150; Dako), CD79a (1:50; Dako), CD3 (1:150; Dako), CD7 (1:150; Dako), and Ki-67 (1:100; Dako) were also added to the samples. Antibodies were used in accordance with manufacturer protocols.

The LNA-ISH and immunostaining positive signal were brown yellow and the TMA sections were scored according to cutoff levels for each ISH or immunohistochemical marker. The scoring was done in a highpower field $(\times 400)$, within an area of $0-16 \mathrm{~mm}^{2}$ almost entirely covering the area of each cylinder section. The LNA-ISH and immunohistochemical results were scored using a semi-quantitative method (Allred score) [20]. The scores were based on the intensity of staining and the percentage of positive results. The percentage of positive cells was used for scoring the positivity: none $=0$; $<1 \%=1 ; 1-10 \%=2 ; 10-33 \%=3 ; 34-67 \%=4$; and $>67 \%=5$ ). The intensity of staining was scored as none $=0$; weak $=1$; intermediate $=2$; and strong $=3$ ). The intensity and percentage scores were added to give a final score of $0-8$. According to the final score, the immunostaining expression signal was determined as negative (0-2), weak positive (3-4), positive (5-6), or strong positive (7-8).

Follow-up and statistical methods

The follow-up information contained overall response rate (ORR), overall survival (OS), and progression-free survival (PFS), which were obtained from hospital information systems and the patients or patients' relatives. ORR was calculated from the first day of treatment to disease remission. OS was calculated from the date of pathological diagnosis to death or the last date of follow-up. PFS was calculated from the date of surgery until the date of recurrence. Patient therapy included chemotherapy or radiotherapy, and the response to first-line therapy included progressive disease (PD), stable disease (SD), partial response (PR), or complete remission (CR). Survival time of patients was analyzed.

All statistical analysis were performed using the SPSS version 13.0 (SPSS, Chicago, IL, USA). Data from qRT$\mathrm{PCR}$ are expressed as mean $\pm \mathrm{SD}$. Univariate analysis of OS and PFS was undertaken using Kaplan-Meier methods. Patients still alive were censused at the last known date of contact. The log-rank test was used to compare survival differences between the groups. Cox regression analysis was used for multivariate analysis. Two-sided $P$ values $<0.05$ were considered statistically significant.

\section{Results}

Study groups and clinicopathological features

Clinical characteristics of the 64 cases of MALT lymphoma and 58 cases of DLBCL analyzed in this study are shown in Table 1 . Lactate dehydrogenase (LDH) and $\beta 2$-microglobulin levels were significantly higher in patients with DLBCL than in those with MALT lymphoma. Significantly more patients with DLBCL were in stage III-IV disease than those with MALT lymphoma. When comparing the two diseases in stage III-IV, OS and PFS of DLBCL were significantly lower than in patients with MALT lymphoma. The 
Table 1 Characteristics of the 122 stomach cases of MALT and DLBCL with clinical and histopathological data

\begin{tabular}{|c|c|c|c|}
\hline \multirow{2}{*}{$\begin{array}{l}\text { Characteristics/ } \\
\text { outcome }\end{array}$} & \multicolumn{2}{|c|}{ Number (\%) patients } & \multirow[t]{2}{*}{$P^{\mathrm{a}}$} \\
\hline & $\begin{array}{l}\text { MALT-BCL }{ }^{\mathrm{b}} \\
(n=64)\end{array}$ & $\begin{array}{l}\text { DLBCL }^{b} \\
(n=58)\end{array}$ & \\
\hline \multicolumn{4}{|l|}{ Age (years) } \\
\hline$<60$ & $30(46.9)$ & $23(39.7)$ & \\
\hline$>60$ & $34(53.1)$ & $35(60.3)$ & $>0.05$ \\
\hline \multicolumn{4}{|l|}{ Gender } \\
\hline Male & $38(59.4)$ & $31(53.4)$ & \\
\hline Female & $26(40.6)$ & $27(46.6)$ & $>0.05$ \\
\hline \multicolumn{4}{|l|}{ LDH level } \\
\hline Normal & $44(68.8)$ & $23(39.7)$ & \\
\hline High & $20(31.2)$ & $35(60.3)$ & $<0.01$ \\
\hline \multicolumn{4}{|l|}{$\beta 2-$ MG level } \\
\hline Normal & $48(75.0)$ & $24(41.4)$ & \\
\hline High & $16(25.0)$ & $34(58.6)$ & $<0.01$ \\
\hline \multicolumn{4}{|l|}{ Helicobacter pylori } \\
\hline Negative & $22(34.4)$ & $38(65.5)$ & \\
\hline Positive & $42(65.6)$ & $20(34.5)$ & $<0.01$ \\
\hline \multicolumn{4}{|l|}{ Stage $e^{c}$} \\
\hline I-II & $40(62.5)$ & $19(32.6)$ & \\
\hline III-IV & $24(37.5)$ & $39(67.4)$ & $<0.01$ \\
\hline \multicolumn{4}{|l|}{ OS } \\
\hline Median (months) & 38 & 25 & \\
\hline 5-year $(\%)$ & 68.8 & 43.2 & $<0.01$ \\
\hline \multicolumn{4}{|l|}{ PFS } \\
\hline Median (months) & 21 & 13 & \\
\hline 5 -year $(\%)$ & 52.6 & 32.4 & $<0.05$ \\
\hline
\end{tabular}

MALT-BCL mucosa-associated lymph tissue B-cell lymphoma, $D L B C L$ diffuse large B-cell lymphoma, $L D H$ lactate dehydrogenase, $\beta 2-M G \quad \beta 2$-microglobulin, $O S$ overall survival, $P F S$ progression-free survival

${ }^{a}$ Clinical characteristics were compared using the $\chi^{2}$ test for categorical variables

b World Health Organization classification (Swerdlow et al. [1])

c Ann Arbor Staging System

Helicobacter pylori infection rate was higher in MALT lymphoma patients $(66 \%)$ than in those with DLBCLs (35\%), which were substantially lower than reported in literature data, possibly because we estimated $H$. pylori only using a histological method with toluidine blue staining and false-negative cases were possible.

Reverse transcription and real-time qPCR

miR-34a was detected by qRT-PCR in all 5 normal control samples and the 20 cases of MALT lymphomas and 20 cases of DLBCLs. After normalization with RNU6, the microRNA expression level in different tissues was calculated and compared with the $\Delta \Delta C_{\mathrm{t}}$ method, assuming $100 \%$ PCR

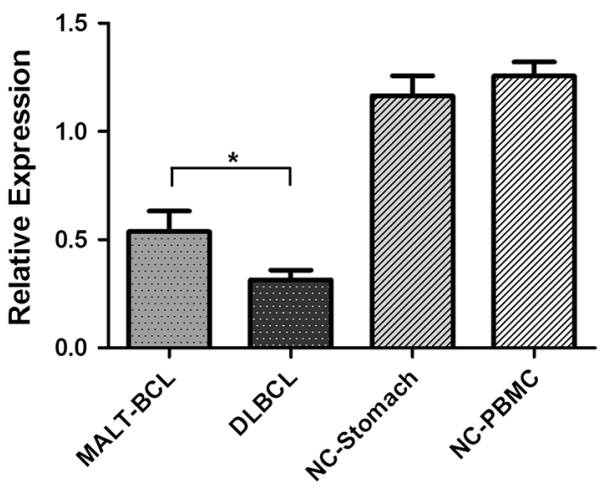

Fig. 1 Quantification of microRNA (miRNA) expression by quantitative real-time reverse transcriptase-polymerase chain reaction (qRTPCR). The levels of miR-34a in gastric mucosa-associated lymphoid tissue (MALT) lymphoma (MALT-BCL, $n=20$ ), diffuse large B-cell lymphoma ( $D L B C L)$ (DLBCL, $n=20$ ), lymphoma normal gastric tissue (NC-Stomach, $n=3$ ), and peripheral blood monocytes $(N C$ $P B M C, n=2$ ) were quantified by qRT-PCR and normalized by RNU6 expression. $y$-axis shows mean relative expression (asterisk). The differentially expressed miR-34a between MALT lymphoma and DLBCL reached statistical significance $(P<0.05)$

efficiency [19]. The results revealed that the miR-34a expression was markedly decreased in DLBCL and MALT lymphoma compared to those in normal gastric tissue and PBMCs (more than twofold lower; $P<0.05$; Fig. 1). In addition, the miR-34a level in DLBCL lymphoma was significantly lower than that in MALT $(P<0.05 ;$ Fig. 1$)$.

Morphological features and miR-34a expression of gastric lymphomas

Prominent lymphoid cells were seen in the mucosa of lymphoma cases in comparison with normal gastric mucosa. The cell population of MALT lymphoma was a mixture of small lymphocytes, plasmacytoid cells, and centrocyte-like lymphocytes. The lymphoma cells had a monocytoid appearance. A characteristic feature of this tumor type was the presence of invasive lymphoepithelial lesions (Fig. 2a). In DLBCL samples, the normal mucosal architecture was replaced by a diffuse infiltrate of large atypical lymphoid tumor cells (Fig. 2b). The tumor cells of both lymphoma types were confirmed to express CD20, indicating a B-cell immunophenotype, and the proliferation index of Ki-67 in MALT lymphoma cells (20-30\%) was lower than in DLBCL cells ( $>50 \%)$.

miR-34a expression was significantly lower in lymphoma tissues than in normal lymph node by the LNA-ISH analysis of 64 cases of MALT lymphoma and 58 cases of DLBCL patients (Fig. 2c). LNA-ISH showed that miR-34a was present in the cytoplasm and nucleus of lymphocytes (Fig. 2c inserted). miR-34a was positive in 16 of the $64(25.0 \%)$ cases of MALT lymphoma and 5 of the $58(8.6 \%)$ cases of DLBCL patients $(P<0.05$; Fig. $3 \mathrm{c}, \mathrm{d})$, and most positive cases were 
Fig. 2 Tissue microarray-based in situ hybridization (ISH) and immunochemistry (IHC) analysis of miR-34a, forkhead box protein 1 (FOXP1), p53, and BCL2 expression in serial sections of gastric MALT lymphoma and DLBCL specimens. Hematoxylin and eosin (H\&E) staining of MALT lymphoma (a) and DLBCL (b). A weak signal for miR-34a was seen in MALT lymphoma (c; insert is lymph node control) and DLBCL tumor cells (d). A stronger signal for FOXP1 was seen in MALT lymphoma (e) and DLBCL tumor cells (f). A weak signal for $\mathrm{p} 53$ was seen in MALT lymphoma cells (g). A stronger signal for $\mathrm{p} 53$ was seen in DLBCL tumor cells (h). MALT lymphoma had strong staining for BCL2 (i). A weak signal for BCL2 was seen in DLBCL cells (j). Browncolored cells are positive signal. $\times 400$

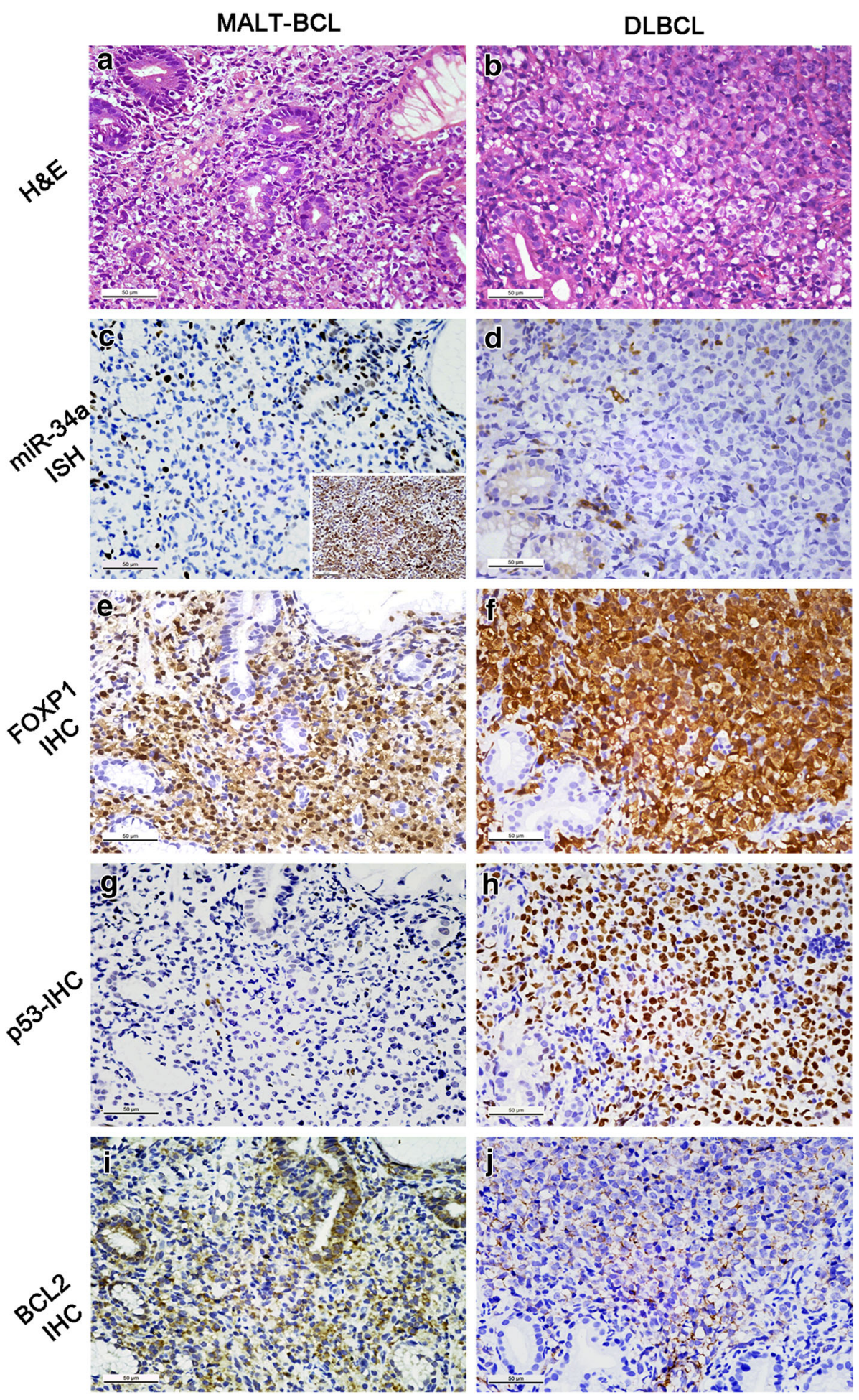

weakly positive. ISH score (Allred score) showed that the samples of gastric MALT lymphoma had a higher positive score for miR-34a (Fig. 3a). The relationship of clinical characteristics with miR-34a expression in lymphoma tissues, as determined by LNA-ISH, is presented in Table 2. The expression of miR-34a is associated with age, $H$. pylori infection, and low (early) disease stage (Table 2).
FOXP1, p53, and BCL2 staining in gastric lymphoma tissues

Figure 2 shows a representation of FOXP1, p53, and BCL2 protein expression in biopsy samples of gastric MALT lymphomas and DLBCLs. Of the 64 MALT lymphoma samples, $31 \%$ were FOXP1 positive (negative 44, weak 

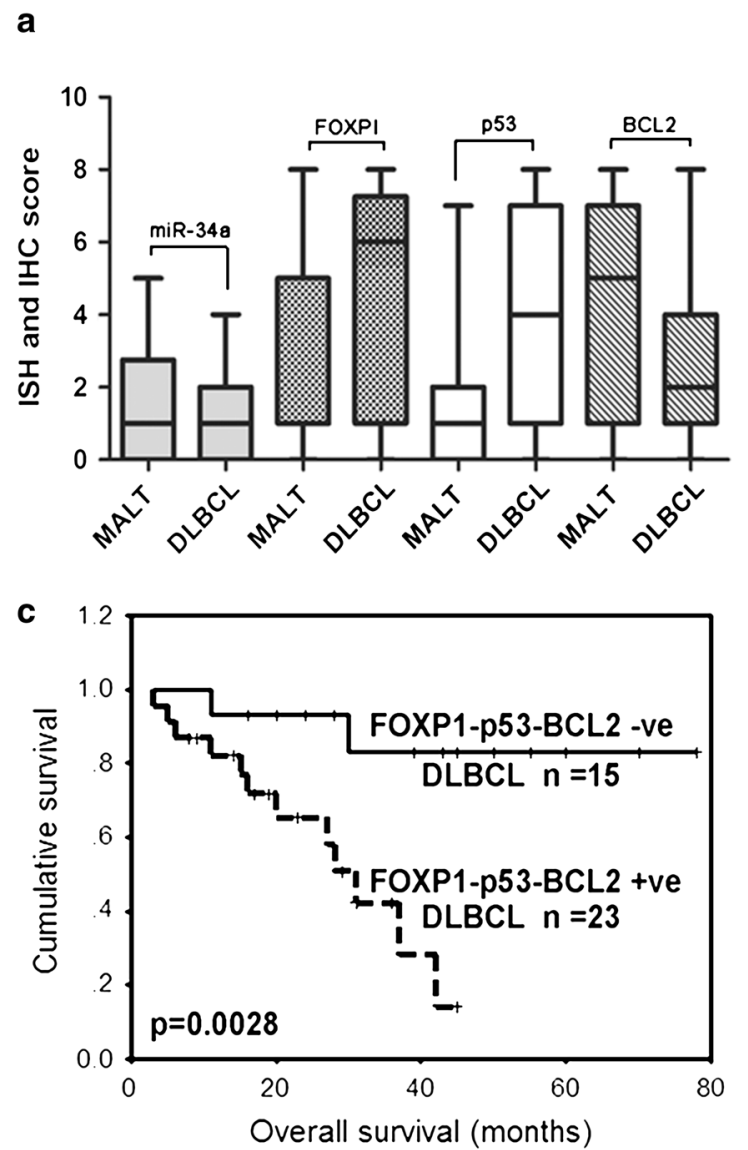

Fig. 3 Locked nucleic acid (LNA) in situ hybridization (ISH) and immunohistochemistry (IHC) score (Allred score) and impact on the prognosis of FOXP1, p53, and BCL2 protein expression in gastric MALT lymphoma and DLBCL patients. a Samples of gastric DLBCL had a higher positive score for FOXP1 and p53; MALT lymphoma samples had a higher positive score for miR-34a and BCL2. Overall

positive 2 , positive 12 , and strong positive 6 ), $19 \%$ were p53 positive (negative 52, weak positive 4 , positive 7 , and strong positive 1 ) and $67 \%$ were BCL2 positive (negative 21 , weak positive 6 , positive 12 , and strong positive 25 ). Among the 58 DLBCL samples, $64 \%$ were FOXP1 positive (negative 21 , weak positive 4 , positive 6 , and strong positive 27), $57 \%$ were p53 positive (negative 25 , weak positive 6 , positive 10 , and strong positive 17 ) and $41 \%$ were BCL2 positive (negative 34, weak positive 11, positive 6 , and strong positive 7). Immunostaining score (Allred score) showed that the samples of gastric DLBCL had a higher positive score for FOXP1 and p53 whereas MALT lymphoma samples had a higher positive score for BCL2 (Fig. 3a). Because sometimes BCL2 expression is variable in MALT lymphomas, it is positive outside colonized follicles but is lost among neoplastic B cells that have colonized reactive follicles. Thus, we calculated BCL2 expression focusing on the neoplastic cells outside the follicles of marginal area and in MALT lymphomas.
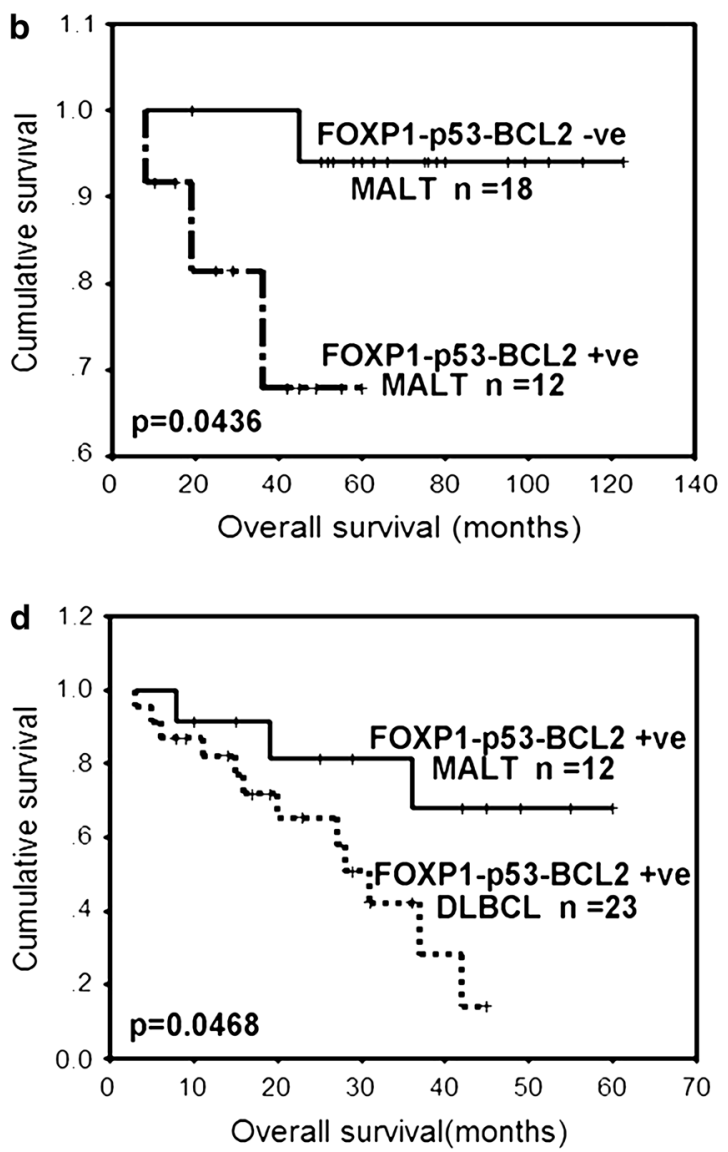

survival (OS) was associated with expression of all FOXP1, p53, and BCL2 in poor prognosis patients with MALT lymphoma $(P=0.044)$ (b) or DLBCL $(P=0.003)(\mathbf{c})$. d Patients with FOXP1-, p53-, and BCL2-positive DLBCL showed significantly worse survival than patients with FOXP1-, p53-, and BCL2-positive MALT lymphomas $(P=0.047)$

Table 2 shows the relative expression of FOXP1, p53, and BCL2, patient age, baseline LDH, $\beta 2$-microglobulin levels, H. pylori infection, and tumor stage.

We compared these patients' clinical parameters with the expression of FOXP1, p53, and BCL2 between the two different types of lymphomas. Analysis indicates that the expression of FOXP1 and p53 is associated with age, LDH, and $\beta 2 \mathrm{M}$ level and with high (advanced) disease stage; BCL2 is associated with H. pylori infection and low (earlier) disease stage (Table 2).

Follow-up and survival

All 122 patients had undergone gastrectomy as the initial treatment. In 64 cases of MALT lymphoma, 4 patients were treated by total gastrectomy and 60 by partial gastrectomy (distal 42, proximal 13, local resection 5). In 58 cases of MALT lymphoma, 16 patients were treated by 
Table 2 Univariate analysis of expression of miR-34a, FOXP1, p53, and BCL2 with clinical characteristics in 64 cases of MALT-BCL and 58 cases of DLBCL in the stomach*

\begin{tabular}{|c|c|c|c|c|c|c|c|c|}
\hline \multirow[t]{2}{*}{ Characteristics/histological type } & \multicolumn{8}{|c|}{ Number (\%) patients } \\
\hline & $\operatorname{miR}-34 a$ & $P$ & FOXP1 & $P$ & p53 & $P$ & BCL2 & $P$ \\
\hline \multicolumn{9}{|l|}{ Age $(>60)$} \\
\hline MALT-BCL & $11(17.2)$ & & $7(10.9)$ & & $10(15.6)$ & & $19(29.7)$ & \\
\hline DLBCL & $1(1.7)$ & $<0.01$ & $20(34.5)$ & $<0.01$ & $23(39.7)$ & $<0.01$ & $13(22.4)$ & $>0.05$ \\
\hline \multicolumn{9}{|l|}{ Gender (male) } \\
\hline MALT-BCL & $8(12.5)$ & & $12(18.8)$ & & $7(10.9)$ & & $22(34.4)$ & \\
\hline DLBCL & $3(5.2)$ & $>0.05$ & $13(22.4)$ & $>0.05$ & $14(24.1)$ & $>0.05$ & $13(22.4)$ & $>0.05$ \\
\hline \multicolumn{9}{|l|}{ LDH level (high) } \\
\hline MALT-BCL & $10(15.6)$ & & $7(10.9)$ & & $3(4.7)$ & & $13(20.3)$ & \\
\hline DLBCL & $2(3.4)$ & $>0.05$ & $28(37.9)$ & $<0.01$ & $24(41.4)$ & $<0.01$ & $16(27.6)$ & $>0.05$ \\
\hline \multicolumn{9}{|l|}{$\beta 2 \mathrm{M}$ level (high) } \\
\hline MALT-BCL & $6(9.4)$ & & $12(18.8)$ & & $7(10.9)$ & & $18(28.1)$ & \\
\hline DLBCL & $3(5.2)$ & $>0.05$ & $30(51.7)$ & $<0.01$ & 27 (46.6) & $<0.01$ & $17(29.3)$ & $>0.05$ \\
\hline \multicolumn{9}{|l|}{ H. pylori $(+)$} \\
\hline MALT-BCL & $12(18.8)$ & & $11(17.2)$ & & $6(10.3)$ & & $20(31.3)$ & \\
\hline DLBCL & $3(5.2)$ & $<0.05$ & $6(10.3)$ & $>0.05$ & $5(8.6)$ & $>0.05$ & $5(8.6)$ & $<0.01$ \\
\hline \multicolumn{9}{|l|}{ Stage I-II } \\
\hline MALT-BCL & $9(14.1)$ & & $8(12.5)$ & & $4(6.3)$ & & $19(29.7)$ & \\
\hline DLBCL & $2(3.4)$ & $<0.05$ & $4(6.9)$ & $>0.05$ & $3(5.2)$ & $>0.05$ & $5(8.6)$ & $<0.01$ \\
\hline \multicolumn{9}{|l|}{ Stage III-IV } \\
\hline MALT-BCL & 7 (10.9) & & $12(18.8)$ & & $8(12.5)$ & & $24(37.5)$ & \\
\hline DLBCL & $1(1.7)$ & $>0.05$ & $33(56.9)$ & $<0.01$ & $29(50.0)$ & $<0.01$ & $19(32.8)$ & $>0.05$ \\
\hline
\end{tabular}

MALT-BCL mucosa-associated lymph tissue B-cell lymphoma, DLBCL diffuse large B-cell lymphoma

* Chi squared test

total gastrectomy and 42 by partial gastrectomy (distal 31 , proximal 11 , local resection 0 ).

The median overall duration of follow-up was 63 months (range, 3-123 months). Eleven (17.2\%) patients with MALT lymphoma and $25(43.1 \%)$ with DLBCL died of lymphoma, treatment toxicity, or unrelated causes; other patients were disease free during the followup period. Among 64 MALT lymphoma patients, of the 42 who were $H$. pylori-positive cases, 38 patients were initially treated by routine $H$. pylori eradication, 30 patients were treated with rituximab combined CHOP chemotherapy after gastrectomy, 19 patients were treated with routine CHOP chemotherapy after gastrectomy, and 15 patients were not treated with chemotherapy or other treatment after gastrectomy. Specifically, 27 of 30 rituximab combined CHOP chemotherapy and 32 patients initially treated by routine $H$. pylori eradication were alive during a median follow-up period of 63 months. The 5-year OS rate for MALT lymphoma (64\%) was substantially lower than that reported in previous studies [21] because some of patients in this study did not receive standard treatment or were not treated with rituximab.
Among 58 DLBCL patients, 20 cases were $H$. pylori positive: 13 patients were initially treated by routine $H$. pylori eradication, 27 patients were treated with rituximab combined CHOP chemotherapy after gastrectomy, 25 patients were treated with routine CHOP chemotherapy after gastrectomy, and 6 patients were not treated with chemotherapy or other treatment after gastrectomy. Specifically, 12 of 27 rituximab combined CHOP chemotherapy and 10 patients initially treated by routine $H$. pylori eradication were alive during a median follow-up period of 38 months.

CR after first-line treatment with therapy occurred in 33 cases $(51.6 \%)$ of MALT lymphoma and 20 cases (34.5\%) of DLBCL. PR was observed in 20 cases $(31.2 \%)$ of MALT lymphoma and 13 cases (22.8\%) of DLBCL. ORR was $82.8 \%$ of MALT lymphoma patients and $56.9 \%$ of DLBCL patients. PD occurred in $15(19.7 \%)$ patients with MALT lymphoma and in $29(50.0 \%)$ patients with DLBCL. The 5-year OS was $68.8 \%$ for MALT lymphoma and $43.2 \%$ for DLBCL (Table 1). Median survival was 38 months (range, 16-60 months) with MALT lymphoma and 25 months (range, 2-48 months) for DLBCL. Median 
PFS was 21 and 13 months, respectively, and the corresponding 5-year PFS rates were $52.6 \%$ and $32.4 \%$ (Table 1).

As expected, patients with advanced-stage disease were more likely to suffer relapses and have a shorter survival time. The expression of miR-34a by LNA-ISH had no significance with OS of gastric MALT lymphoma or DLBCL patients, respectively, as the low positive rate. FOXP1, p53, and BCL2 by immunohistochemistry had no significance with OS of gastric MALT lymphoma or DLBCL patients, respectively. Additional analysis indicated that combined expression of FOXP1, p53, and BCL2 in MALT lymphoma $(n=12)$ and DLBCL $(n=23)$ were associated with reduced overall survival $(P=0.044$ and 0.003 , respectively; Fig. 3b, c). Also, FOXP1, p53, and BCL2 coexpressed cases were negative with miR-34a LNA-ISH. DLBCL patients who were FOXP1-, p53-, and BCL2-positive $(n=23)$ showed a significantly worse survival rate than patients with FOXP1-, p53-, and BCL2positive MALT lymphomas $(P=0.047$; Fig. 3d). KaplanMeier OS curves stratifying patients according to FOXP1, p53, and BCL2 protein expression are shown in Fig. 3.

Univariate analysis of expression of miR-34a, FOXP1, p53, and BCL2 with clinical characteristics in 64 cases of

Table 3 Univariate analysis associated with PFS and OS in 64 cases of MALT-BCL and 58 cases of DLBCL in stomach*

\begin{tabular}{|c|c|c|c|c|c|c|c|c|c|c|}
\hline \multirow{2}{*}{$\begin{array}{l}\text { Characteristics/ } \\
\text { outcome }\end{array}$} & \multicolumn{5}{|c|}{ MALT-BCL $(n=64)$} & \multicolumn{5}{|l|}{$\operatorname{DLBCL}(n=58)$} \\
\hline & Patient numbers & $\begin{array}{l}\text { PFS (m) } \\
\text { Median }\end{array}$ & $P$ & $\begin{array}{l}\text { OS (m) } \\
\text { Median }\end{array}$ & $P$ & Patient numbers & $\begin{array}{l}\text { PFS (m) } \\
\text { Median }\end{array}$ & $P$ & $\begin{array}{l}\text { OS (m) } \\
\text { Median }\end{array}$ & $P$ \\
\hline \multicolumn{11}{|l|}{ Age (years) } \\
\hline$<60$ & 30 & 36.2 & & 32.8 & & 23 & 23.2 & & 14.8 & \\
\hline$>60$ & 34 & 23.8 & 0.372 & 21.3 & 0.458 & 35 & 12.3 & 0.758 & 10.4 & 0.252 \\
\hline \multicolumn{11}{|l|}{ Gender } \\
\hline Male & 38 & 39 & & 34.7 & & 31 & 21.7 & & 13.7 & \\
\hline Female & 26 & 28.1 & 0.405 & 20.4 & 0.554 & 27 & 11.4 & 0.654 & 9.8 & 0.008 \\
\hline \multicolumn{11}{|l|}{ LDH level } \\
\hline Normal & 44 & 44.7 & & 33 & & 23 & 35.8 & & 30.5 & \\
\hline High & 20 & 15.3 & 0.016 & 13.5 & 0.084 & 35 & 10.7 & 0.074 & 5.7 & 0.003 \\
\hline \multicolumn{11}{|l|}{$\beta 2 \mathrm{M}$ level } \\
\hline Normal & 48 & 45.7 & & 31.5 & & 24 & 32 & & 22.9 & \\
\hline High & 16 & 17.4 & 0.004 & 18.9 & 0.022 & 34 & 13.4 & 0.114 & 10.6 & 0.015 \\
\hline \multicolumn{11}{|l|}{ H. pylori } \\
\hline Negative & 22 & 48.6 & & 38 & & 38 & 35.1 & & 31.6 & \\
\hline Positive & 42 & 16.2 & 0.048 & 12.4 & 0.031 & 20 & 10.9 & 0.246 & 7 & 0.094 \\
\hline \multicolumn{11}{|l|}{ Stage } \\
\hline I-II & 40 & 46.7 & & 38.1 & & 19 & 34.8 & & 19.8 & \\
\hline III-IV & 24 & 15.4 & 0.061 & 11.8 & 0.005 & 39 & 9.4 & 0.003 & 8.4 & 0.025 \\
\hline \multicolumn{11}{|l|}{$\operatorname{miR}-34 a$} \\
\hline Negative & 46 & 17.6 & & 13.7 & & 53 & 11.7 & & 6.3 & \\
\hline Positive & 16 & 42 & 0.001 & 38.9 & 0.007 & 5 & 33.9 & 0.009 & 28.6 & 0.024 \\
\hline \multicolumn{11}{|l|}{ FOXP1 } \\
\hline Negative & 44 & 40.1 & & 33.7 & & 21 & 31 & & 29.4 & \\
\hline Positive & 20 & 16.3 & 0.042 & 8.5 & 0.002 & 37 & 14.3 & 0.027 & 5.5 & 0.008 \\
\hline \multicolumn{11}{|l|}{ p53 } \\
\hline Negative & 52 & 38 & & 35.6 & & 25 & 30.7 & & 28.8 & \\
\hline Positive & 12 & 20 & 0.245 & 16 & 0.082 & 33 & 13.4 & 0.019 & 11.1 & 0.002 \\
\hline \multicolumn{11}{|l|}{ BCL2 } \\
\hline Negative & 21 & 45.5 & & 40.9 & & 34 & 36.8 & & 34.9 & \\
\hline Positive & 43 & 31.4 & 0.986 & 22.1 & 0.645 & 24 & 23.5 & 0.546 & 19.4 & 0.378 \\
\hline
\end{tabular}

$M A L T-B C L$ mucosa-associated lymph tissue B cell-lymphoma, $D L B C L$ diffuse large B-cell lymphoma, $L D H$ lactate dehydrogenase, $\beta 2-M G \beta 2$ microglobulin, $O S$ overall survival, $P F S$ progression-free survival

* Univariate analysis by Kaplan-Meier test 
Table 4 Multivariate survival analysis (Cox regression model) of conventional clinical prognostic factors and miR-34a, FOXP1, p53, and BCL2 expression in 122 stomach cases of MALT and DLBCL

\begin{tabular}{llll}
\hline Variables & $\begin{array}{l}\text { Hazard } \\
\text { ratio }\end{array}$ & $\begin{array}{l}\text { 95\% Confidence } \\
\text { intervals }\end{array}$ & $P$ value \\
\hline miR-34a positive & 2.287 & $1.108-4.754$ & 0.043 \\
FOXP1 positive & 0.513 & $0.252-1.036$ & 0.067 \\
p53 positive & 2.264 & $0.973-5.268$ & 0.058 \\
BCL2 positive & 1.093 & $0.692-1.728$ & 0.702 \\
FOXP1-p53-BCL2 & 2.349 & $1.119-4.854$ & 0.027 \\
$\quad$ positive & & & \\
FOXP1-p53-BCL2 & 0.865 & $0.515-1.455$ & 0.586 \\
$\quad$ negative & & & \\
Age & 1.212 & $0.760-1.931$ & 0.420 \\
LDH level & 2.829 & $1.181-6.869$ & 0.023 \\
B2-MG level & 0.561 & $0.227-1.476$ & 0.253 \\
H. pylori & 1.268 & $0.771-2.085$ & 0.350 \\
Stage & 4.033 & $1.842-8.895$ & 0.001 \\
\hline
\end{tabular}

MALT-BCL and 58 cases of DLBCL are shown in Table 2. Prognosis of univariate analysis associated with PFS and OS in 64 cases of MALT-BCL and 58 cases of DLBCL are shown in Table 3. Multivariate survival analysis (Cox regression model) of conventional clinical prognostic factors and miR-34a, FOXP1, p53, and BCL2 expression in 122 stomach cases of MALT and DLBCL showed coexpression of FOXP1, p53, and BCL2 was an independent prognostic factor in Cox regression multivariate survival analysis (95 \% CI, 1.119-4.854; $P=0.027$ ). The miR-34a (95\% CI, 1.108-4.754; $P=0.043)$ and stage (III + IV) (95\% CI, 1.842-8.895; $P=0.001)$ also showed significance (Table 4$)$.

\section{Discussion}

miR-34a has multiple experimentally validated targets that are involved in cellular proliferation and apoptosis. Thus, the level of miR-34a expression is thought to regulate the balance between apoptosis and cell-cycle arrest [6, 7]. The human miR-34a precursor is transcribed from chromosome 1. It maps to the distal region of chromosome $1 \mathrm{p}$, which is a fragile chromosomal region commonly deleted in lymphoma cells, especially in B-cell lymphomas [7]. Primary gastric lymphomas, predominantly B-lymphocyte forms of non-Hodgkin lymphoma (NHL), has been identified as the second most common form of stomach malignant tumors, which are commonly classified as MALT lymphoma and DLBCL. Both forms of the lymphomas have definitive clinicopathological entities characterized by their unique histological and clinical features [1-3]. Research showed that miR-34a regulation of B-cell development and apoptosis were possibly related to the tumor suppressor p53, oncogenic protein BCL2, and FOXP1 [10-18]. In this study, we assessed corelations of miR-34a level and the expression of the B-cell differentiation- and apoptosisrelated proteins FOXP1, p53, and BCL2 with the clinicopathological features and survival of gastric MALT lymphoma and DLBCL.

In this analysis, using qRT-PCR, we showed that miR$34 \mathrm{a}$ expression was markedly decreased in DLBCL and MALT lymphoma cells compared to normal gastric tissue and PBMC. The level of miR-34a was much lower in DLBCL than MALT lymphoma, which was in part as in a previous study [16]. Next, by the LNA-ISH analysis of 64 cases of MALT lymphoma and 58 cases of DLBCL tissues, we found that the miR-34a expression was significantly lower in lymphoma than in normal lymph node. Although the miR-34a expression in the two types of gastric lymphomas was significant, there were no statistically significant differences with OS in the two types of gastric lymphomas, possibly because of the low positive rate, Then, we detected the miR-34a target B-cell differentiation- and apoptosis-related proteins FOXP1, p53, and BCL2 expression immunochemically in lymphoma tissues. No statistically significant differences were seen with OS in the two types of gastric lymphomas; however, overall survival analysis indicated that combined expression of FOXP1, p53, and BCL2 in MALT lymphoma and DLBCL were associated with reduced survival time, and patients with tumors that coexpressed FOXP1, p53, and BCL2 had a poor OS regardless of lymphoma type. Furthermore, FOXP1-, p53-, and BCL2-positive DLBCL tumors had a significantly worse survival rate than corresponding MALT lymphomas. Specifically, multivariate Cox regression analysis showed miR-34a down-expression, coexpression of FOXP1, p53, and BCL2, and advanced disease (stage III and IV) were independent prognostic factors, i.e., miR-34a down-expression and high FOXP1, p53, and BCL2 expression in advanced-stage disease (III + IV) had a significant adverse impact on survival.

In literature reports, it is well known that miRNAs can influence lineage choice or affect critical developmental checkpoints during hematopoiesis [6]. Constitutive expression of miR-34a was reported to block B-cell development at the pro-B-cell to pre-B-cell transition stage, resulting in a reduction in mature $B$ cells [7]. This blockage appeared to be mediated primarily by inhibiting the expression of the transcription factor FOXP1 [15, 16, 22]. FOXP1 is therefore, thought to be an essential participant in the transcriptional regulatory network responsible for B lymphopoiesis and is needed for B-cell differentiation and life-cycle management [16, 22]. Several studies have indicated that the expression of FOXP1 in DLBCL is associated with poor clinical outcome [16, 17, 
23-25]. Previous studies with MALT lymphoma indicate that FOXP1 positivity is confined to MALT lymphomas with poor clinical outcome, polymorphic histology, and high risk of transforming into DLBCL [18, 26, 27]. The previously described role of miR-34a is as a direct link between the tumor suppressor p53 and the oncogenic protein BCL2 [13, 14]. Yu et al. found FOXP1 was positively correlated with the expression of BCL2 mainly in the non-germinal center B cell subgroup of DLBCL cells. FOXP1 was also predominantly expressed in a similar subset of DLBCL with positive BCL2 [25]. BCL2 protein overexpression has been reported to occur in 24-66 \% of cases of DLBCL $[26,27]$. It has been postulated that in DLBCL changes in FOXP1 and BCL2 were interdependent and that the presence of an additional copy of FOXP1 gene will cause BCL2 protein to be active [28, 29]. The role of miR-34a as a tumor suppressor may be more directly related to $\mathrm{p} 53$ and BCL2. An additional regulation point in B-cell development has been identified on the miR-34aFOXP1 transcription factor axis [10, 11, 13, 14, 22]. Following inactivation of miR-34a, its effect on the B-cell developmental pathway is consistent with the abnormalities seen with FOXP1, p53, and BCL2 expression, which influence the development of mature $\mathrm{B}$ cells and promote malignant transformation associated with B-cell lymphomas $[22,30]$.

It has been proposed that miRNAs may be potential biomarkers for tumor diagnosis, prognosis, and targets for therapy [31]. MALT lymphoma and DLBCL are the two most common types of gastric lymphomas, which have different clinicopathological features with distinct clinical outcomes. Our results indicate that miR-34a expression is significantly decreased in gastric MALT lymphoma and DLBCL. Interestingly, FOXP1, p53, and BCL2 coexpressed cases were negative with miR-34a expression and a poor OS. FOXP1-, p53-, and BCL2-positive DLBCL patients had an even lower OS than corresponding MALT lymphoma patients. What are the mechanisms or reasons that such down-expression and coexpression of these biomarkers affected the prognosis? It may be associated with the following points. (1) miR-34a is a tumor suppressor that connects the p53 network through FOXP1 and BCL2. Each point in this pathway network determines the regulation of apoptosis and development of a mature B cell and is associated with malignant transformation of B-cell lymphomas. (2) The prime mechanism of tumor suppressor miR-34a in gastric MALT lymphoma and DLBCL is downregulated, and down-expression of miR-34a causes the high expression of p53, FOXP1, and BCL2, which leads to the abnormal development, proliferation, and apoptosis of mature B-cell lymphocyte and is related to the tumorigenesis of lymphomas. (3) Down-expression of miR-34a and coexpression of p53, FOXP1, and BCL2 in gastric MALT lymphoma and DLBCL are correlated to the poor prognosis, implying the important role of the miR-34a network.

In a word, decreased miR-34a expression and increased FOXP1, p53, and BCL2 coexpression predict a poor OS for stomach MALT lymphoma and DLBCL patients. We propose that the changes and prognostic significance of miR-34a and its target proteins FOXP1, p53, and BCL2 in gastric MALT lymphoma and DLBCL warrant further investigation as potential prognostic markers that could be potentially used in routine pathology observation.

Acknowledgments This study was supported by Ministry of Health special scientific fund for non-profit public industry of China (No. 200802011), and the National Natural Science Foundation of China (Project No. 81372544 and 81172249).

Conflict of interest The authors declare that they have no competing interests.

\section{References}

1. Swerdlow SH, Campo E, Harris NL, Jaffe ES, Pileri SA, Stein H, Thiele J, Vardiman JW. World Health Organization classification of tumours: pathology and genetics of tumours of haematopoietic and lymphoid tissues. Lyon: IARC; 2008.

2. Howell JM, Auer-Grzesiak I, Zhang J, Andrews CN, Stewart D, Urbanski SJ. Increasing incidence rates, distribution and histological characteristics of primary gastrointestinal non-Hodgkin lymphoma in a North American population. Can J Gastroenterol. 2012;26:452-6.

3. Alevizos L, Gomatos IP, Smparounis S, Konstadoulakis MM, Zografos G. Review of the molecular profile and modern prognostic markers for gastric lymphoma: how do they affect clinical practice? Can J Surg. 2012;55:117-24.

4. Tzankov A, Zlobec I, Went P, Robl H, Hoeller S, Dirnhofer S. Prognostic immunophenotypic biomarker studies in diffuse large B cell lymphoma with special emphasis on rational determination of cut-off scores. Leuk Lymphoma. 2010;51:199-212.

5. Lim LP, Glasner ME, Yekta S, Burge CB, Bartel DP. Vertebrate microRNA genes. Science. 2003;299:1540.

6. Chen CZ, Li L, Lodish HF, Bartel DP. MicroRNAs modulate hematopoietic lineage differentiation. Science. 2004;303:83-6.

7. Hermeking H. The miR-34 family in cancer and apoptosis. Cell Death Differ. 2010;17:193-9.

8. He L, He X, Lim LP, de Stanchina E, Xuan Z, Liang Y, Xue W, Zender L, Magnus J, Ridzon D, Jackson AL, Linsley PS, Chen C, Lowe SW, Cleary MA, Hannon GJ. A microRNA component of the p53 tumour suppressor network. Nature (Lond). 2007;447:1130-4.

9. Bommer GT, Gerin I, Feng Y, Kaczorowski AJ, Kuick R, Love RE, Zhai Y, Giordano TJ, Qin ZS, Moore BB, MacDougald OA, Cho KR, Fearon ER. p53-mediated activation of miRNA34 candidate tumor-suppressor genes. Curr Biol. 2007;17:1298-307.

10. Chang TC, Wentzel EA, Kent OA, Ramachandran K, Mullendore M, Lee KH, Feldmann G, Yamakuchi M, Ferlito M, Lowenstein CJ, Arking DE, Beer MA, Maitra A, Mendell JT. Transactivation of miR-34a by p53 broadly influences gene expression and promotes apoptosis. Mol Cell. 2007;26:745-52.

11. Ji Q, Hao X, Meng Y, Zhang M, Desano J, Fan D, Xu L. Restoration of tumor suppressor miR-34 inhibits human p53-mutant gastric cancer tumor spheres. BMC Cancer. 2008;8:266. 
12. Dijkstra MK, van Lom K, Tielemans D, Elstrodt F, Langerak AW, van't Veer MB, Jongen-Lavrencic M. 17p13/TP53 deletion in B-CLL patients is associated with microRNA-34a downregulation. Leukemia. 2009;23:625-7.

13. Wang X, Liu P, Zhu H, Xu Y, Ma C, Dai X, Huang L, Liu Y, Zhang L, Qin C. miR-34a, a microRNA up-regulated in a double transgenic mouse model of Alzheimer's disease, inhibits BCL2 translation. Brain Res Bull. 2009;80:268-73.

14. Tarasov V, Jung P, Verdoodt B, Lodygin D, Epanchintsev A, Menssen A, Meister G, Hermeking H. Differential regulation of microRNAs by 553 revealed by massively parallel sequencing: miR-34a is a p53 target that induces apoptosis and G1-arrest. Cell Cycle. 2007;6:1586-93.

15. Hu H, Wang B, Borde M, Nardone J, Maika S, Allred L, Tucker PW, Rao A. FOXP1 is an essential transcriptional regulator of B cell development. Nat Immunol. 2006;7:819-26.

16. Craig VJ, Cogliatti SB, Imig J, Renner C, Neuenschwander S, Rehrauer H, Schlapbach R, Dirnhofer S, Tzankov A, Müller A. myc-mediated repression of microRNA-34a promotes high-grade transformation of B-cell lymphoma by dysregulation of FOXP1. Blood. 2011;117:6227-36.

17. Barrans SL, Fenton JA, Banham A, Owen RG, Jack AS. Strong expression of FOXP1 identifies a distinct subset of diffuse large B-cell lymphoma (DLBCL) patients with poor outcome. Blood. 2004;104:2933-5.

18. Han SL, Wu XL, Wan L, Zeng QQ, Li JL, Liu Z. FOXP1 expression predicts polymorphic histology and poor prognosis in gastric mucosa-associated lymphoid tissue lymphomas. Dig Surg. 2009;26:156-62.

19. Livak KJ, Schmittgen TD. Analysis of relative gene expression data using real-time quantitative PCR and the 2(-Delta Delta C(T)) Method. Methods. 2001;25:402-8.

20. Allred DC, Clark GM, Elledge R, Fuqua SA, Brown RW, Chamness GC, Osborne CK, McGuire WL. Association of p53 protein expression with tumour cell proliferation rate and clinical outcome in node-negative breast cancer. J Natl Cancer Inst. 1993;85:200-6.

21. Koch P, del Valle F, Berdel WE, Willich NA, Reers B, Hiddemann W, Grothaus-Pinke B, Reinartz G, Brockmann J, Temmesfeld A, Schmitz R, Rübe C, Probst A, Jaenke G, Bodenstein H, Junker A, Pott C, Schultze J, Heinecke A, Parwaresch R, Tiemann M, German Multicenter Study Group. Primary gastrointestinal non-Hodgkin's lymphoma: I. Anatomic and histologic distribution, clinical features, and survival data of 371 patients registered in the German Multicenter Study GIT NHL 01/92. J Clin Oncol. 2001;19:3861-73.
22. Hoeller S, Schneider A, Haralambieva E, Dirnhofer S, Tzankov A. FOXP1 protein overexpression is associated with inferior outcome in nodal diffuse large B-cell lymphomas with non-germinal centre phenotype, independent of gains and structural aberrations at 3p14.1. Histopathology. 2010;57:73-80

23. Brown PJ, Ashe SL, Leich E, Burek C, Barrans S, Fenton JA, Jack AS, Pulford K, Rosenwald A, Banham AH. Potentially oncogenic B-cell activation-induced smaller isoforms of FOXP1 are highly expressed in the activated B cell-like subtype of DLBCL. Blood. 2008;111:2816-24.

24. Yu B, Zhou X, Li B, Xiao X, Yan S, Shi D. FOXP1 expression and its clinicopathologic significance in nodal and extranodal diffuse large B-cell lymphoma. Ann Hematol. 2011;90:701-8.

25. Sagaert X, de Paepe P, Libbrecht L, Vanhentenrijk V, Verhoef G, Thomas J, Wlodarska I, De Wolf-Peeters C. Forkhead box protein $\mathrm{P} 1$ expression in mucosa-associated lymphoid tissue lymphomas predicts poor prognosis and transformation to diffuse large B-cell lymphoma. J Clin Oncol. 2006;24:2490-7.

26. Kusumoto S, Kobayashi Y, Sekiguchi N, Tanimoto K, Onishi Y, Yokota Y, Watanabe T, Maeshima AM, Ishida T, Inagaki H, Matsuno Y, Ueda R, Tobinai K. Diffuse large B-cell lymphoma with extra BCL-2 gene signals detected by FISH analysis is associated with a "non-germinal center phenotype". Am J Surg Pathol. 2005;29:1067-73.

27. Iqbal J, Neppalli VT, Wright G, Dave BJ, Horsman DE, Rosenwald A, Lynch J, Hans CP, Weisenburger DD, Greiner TC, Gascoyne RD, Campo E, Ott G, Müller-Hermelink HK, Delabie J, Jaffe ES, Grogan TM, Connors JM, Vose JM, Armitage JO, Staudt LM, Chan WC. BCL2 expression is a prognostic marker for the activated B-cell-like type of diffuse large B-cell lymphoma. J Clin Oncol. 2006;24:961-8.

28. Korać P, Dominis M. Prognostic markers and gene abnormalities in subgroups of diffuse large B-cell lymphoma: single center experience. Croat Med J. 2008;49:618-24.

29. Korać P, Vintar MG, Ajduković R, Kardum Paro MM, Jaksić B, Dominis M. FOXP1 and BCL2 show similar immunoenzymatic pattern in bone marrow trephines of chronic lymphocytic leukemia patients. Appl Immunohistochem Mol Morphol. 2009;17: 500-4.

30. Belver L, Papavasiliou FN, Ramiro AR. MicroRNA control of lymphocyte differentiation and function. Curr Opin Immunol. 2011;23:368-73.

31. Cho WC. MicroRNAs: potential biomarkers for cancer diagnosis, prognosis and targets for therapy. Int $\mathrm{J}$ Biochem Cell Biol. 2010;42:1273-81. 